\title{
New embedding model of general relativity
}

\author{
S. Deser, ${ }^{*}$ F. A. E. Pirani, and D. C. Robinson \\ Department of Mathematics, King's College, Strand, London W.C.2, England
}

(Received 15 March 1976)

The string-model analog of general relativity is shown to be unphysically dependent on an embedding gauge. Moreover, an explicit example confirms that it is inequivalent to Einstein's theory.

Regge and Teitelboim have developed ${ }^{1}$ a variation of general relativity employing the string model and have discussed certain difficulties associated with it. In this note we shall show that their theory, which we shall call the new embedding model of general relativity, suffers from gauge dependence in a way which appears to be physically unacceptable. We shall show further, by example, that there are vacuum solutions of the ReggeTeitelboim equations which are not vacuum solutions of Einstein's equations, thereby confirming their statement that the physical interpretations of the two theories are different.

A typical string model is an intrinsically $n$-dimensional dynamical system $S$ embedded (locally) in a manifold $M$ of dimension $m>n$; the latter may or may not be flat. The dynamical variables are the "amplitudes" $y^{A}(x), A=1, \ldots, m$, which locate the system in the embedding space $M$, while the coordinates $x^{\mu}, \mu=1, \ldots, n$ parametrize the embedded system $S$. The action is of the form

$$
I=-\int d^{n} x R(g),
$$

where $R$ is any scalar density on $S$ built up from the metric $g$ of $S$; this metric is induced from that of $M$ by the embedding map $S \rightarrow M$. In the string models discussed here $M$ is flat, the $y^{A}$ are (pseudo-) Cartesian coordinates on $M$, and the components of $g$ are given explicitly by

$$
g_{\mu \nu}(x)=\eta_{A B} y^{A}{ }_{, \mu}(x) y^{B}{ }_{, \nu}(x) ;
$$

here and below subscript and superscript commas denote partial differentiation and $\eta_{A B}$ is the pseudoCartesian metric on $M$. By "pseudo-Cartesian" we mean that $\eta$ is diagonal, with entries +1 and -1 . If +1 appears $p$ times and -1 appears $q$ $=m-p$ times, then $M$ is denoted $E^{p, q}$.

For a string model the external variables $y^{A}$, not the $g_{\mu \nu}$, are chosen as field quantities, the density $R(g)$ is written as a function $R(g(y))$ by substituting for the $g$ 's from Eqs. (2), and the field equations are not

$$
0=\delta I / \delta g_{\mu \nu}(x) \equiv \mathcal{S}^{\mu \nu}(x),
$$

but rather

$$
\begin{aligned}
0 & =\delta I / \delta y^{A}(x) \\
& =-2\left[\mathcal{G}^{\mu \nu}(y(x)) y^{A},{ }_{\mu}\right]_{, \nu} .
\end{aligned}
$$

Alternatively, one may take both the $g_{\mu \nu}$ and the $y^{A}$ as field quantities and deduce Eqs. (2), as well as (4), from the variational principle by introducing a Lagrange multiplier field $\lambda^{\mu \nu}$ and substituting for Eq. (1) the action

$$
I=-\int d^{n} x\left[R(g)-\lambda^{\mu \nu}\left(g_{\mu \nu}-\eta_{A B} y^{A}{ }_{\mu} y^{B}{ }_{, \nu}\right)\right] .
$$

With the help of the Noether identities

$$
\nabla_{\nu} \mathcal{g}^{\mu \nu}=0
$$

where $\nabla_{\nu}$ denotes the covariant derivative associated with $g$, one may rewrite the string-model, or external-variables, field equations (4) in the manifestly $S$-covariant form

$$
g^{\mu \nu} \nabla_{\mu} \nabla_{\nu} y^{A}=0 \text {. }
$$

Notice that for any given embedding the $y^{A}$ are scalar functions on $S$. Equations (5) are invariant under the action of the orthogonal group of signature $p, q, \mathrm{O}(p, q)$, on $E^{p, q}$, which produces rigid rotations or pseudorotations of the embedded manifold $S$ in $E^{p, q}$.

As Regge and Teitelboim point out, the identity

$$
\eta_{A B} y^{A}, \lambda \nabla_{\mu} \nabla_{\nu} y^{B}=0,
$$

which follows from $\nabla_{\lambda} g_{\mu \nu}=0$, prevents the inference from (5) that $g^{\mu \nu}=0$, since it shows that the $\nabla_{\mu} \nabla_{\nu} y^{B}$ are not linearly independent.

In the original string model (geometrically, $n$ $=2$ is an exceptional case $\left.^{2}\right), n=2$ and $R=(-g)^{1 / 2}$. In the new embedding model, $n=4$ and $\mathbb{R}$ is the Einstein scalar curvature density. The (vacuum) Euler-Lagrange equations for the new embedding model are equations (5) with $g^{\mu \nu}=(-g)^{1 / 2} G^{\mu \nu}$, where $G^{\mu \nu}$ is the Einstein tensor. If $R$ is replaced by $R$ $-2 \lambda$, where $\lambda$ is a constant, then the EulerLag range equations are again (5), but now with $g^{\mu \nu}=(-g)^{1 / 2}\left(G^{\mu \nu}+\lambda g^{\mu \nu}\right)$. As Regge and Teitelboim point out, and as we confirm explicitly by an example below, these new-embedding-model equa- 
tions are, on account of (6), weaker than, not equivalent to, Einstein's vacuum field equations. It appears to be the derivative character of the change of field variables (2), rather than the nonlinearity, which is responsible for this weakening: Observe that the (nonlinear) substitution $g_{\mu \nu}$ $=\eta_{\alpha \beta} e_{\mu}^{\alpha} e_{\nu}^{\beta}$ embodying the introduction of a vierbein field $e_{\mu}^{\alpha}$ actually increases the number of field variables from 10 to 16 , but the vierbein EulerLagrange equations analogous to (5), namely $(-g)^{1 / 2} G^{\mu \nu} e_{\nu}^{\alpha}=0$, are, in contrast, strictly equivalent to Einstein's vacuum equations.

It is worth mentioning that although the substitution (2) expresses the $g$ 's in terms of the first derivatives of the $y$ 's, wherefore one might expect the field equations, which contain second derivatives of the $g$ 's, to contain third derivatives when written in terms of the $y$ 's, nevertheless, all the third-derivative terms cancel out, so that the newembedding-model field equations are in fact trilinear in the second derivatives of the external variables $y^{A}$. This essential nonlinearity, which is of course related to the geometrical interpretation in terms of embedding, makes it difficult to formulate a linearized theory representing weak excitations of the $y^{A}$ : Heuristically speaking, an assumption of the form $y=x+\epsilon$, where $\epsilon$ is supposed weak, yields, on substitution into Eqs. (2), a metric of the form

$$
\begin{aligned}
g= & \text { flat metric } \\
& + \text { gauge terms removable by } \\
& \text { coordinate transformation } \\
& + \text { terms quadratic in } \epsilon,
\end{aligned}
$$

so that the linearized weak-field approximation $g=\eta+h$ of Einstein's theory is inaccessible in terms of the $y$ 's.

Clarke has shown recently ${ }^{3}$ that global embedding of any Lorentzian 4-manifold $S$ in a flat $M$ is possible with $m=91$ ( $m=50$ if $S$ is compact). Local embeddings are always possible with $m=10,{ }^{4}$ although the local minimal value of $m$ may vary over $S$, and, as is well known and will be shown in the examples below, local embeddings with $m<10$ are possible in particular cases, although a nonflat solution of the vacuum Einstein equations cannot be embedded, even locally, with $m<6$. Regge and Teitelboim postulate $m \geqslant 10$ so as to be able to include, at least locally, the generic case.

For any given manifold $S$, there is an infinity of embeddings $S \rightarrow M$ (with $M$ not necessarily always the same). We call the corresponding transformations of the $y$ 's "changes of embedding gauge," and emphasize that

(a) if an embedding with a particular value of $m$ is given, then any nonsingular linear transforma- tion of the $y$ 's, with constant coefficients, yields another embedding with the same value of $m$, and if, furthermore, both the original and the transformed $y$ 's are pseudo-Cartesian, then the transformation is an element of $\mathrm{O}(p, q)$;

(b) if an embedding with a particular value of $m$ is given, then there may exist a nonlinear transformation of the $y$ 's which yields another pseudoCartesian embedding with the same $p, q$, and $m$; this corresponds to a deformation of the embedded manifold $S$ (we shall give a particular example of a deformation, but we have not worked out which solutions of the vacuum Einstein equations admit such deformations); and

(c) if an embedding with a particular value of $m$ is given, then an embedding with any larger value of $m$ may be constructed, more or less trivially, by introducing supernumerary $y$ 's. For example, one may adjoin $y^{m+1}(x)=y^{m+2}(x)$, with $\eta_{m+1, m+1}$ $=-\eta_{m+2, m+2}=1$. This leaves $g_{\mu \nu}$, and hence all observable phenomena, unaltered, but $y^{m+1}$ may be an arbitrary function on $S$ and if $g^{\mu \nu} \neq 0$, then equation (5) will not in general be satisfied by $y^{m+1}$ or $y^{m+2}$ even if $y^{1}, \ldots, y^{m}$ satisfy it. Thus the new embedding model is unphysically dependent on the embedding gauge.

We now give a concrete example which illustrates some of these points. We exhibit a class $C$ of spacetimes $S$ with the properties:

(1) there is a local embedding $S \rightarrow E^{5,2}$ for each $S$ in $C$, satisfying the new-embedding-model vacuum equations (5);

(2) only those spacetimes in a subclass $C_{0}$ satisfy Einstein's vacuum field equations $g^{\mu \nu}=0$; and

(3) for the spacetimes in a subclass $C_{1}$, disjoint from $C_{0}$, there is a local embedding $S-E^{5,2}$ not satisfying the new-embedding-model vacuum equations (5).

The class $C$ consists of the plane-fronted wave solutions of the Einstein-Maxwell equations ${ }^{5}$ : Let

$$
d s^{2}=2 d u d v+2 H(x, y, u) d u^{2}+d x^{2}+d y^{2},
$$

with (for convenience) $H>0$ in the spacetime domain under consideration. The Einstein tensor is given by

$$
G_{\mu \nu}=u,{ }_{\mu} u_{\nu} \square H,
$$

where $\square H=H_{, x x}+H_{\text {,yy }}$. A straightforward calculation gives

$$
G^{\mu \nu} \nabla_{\mu} \nabla_{\nu} y^{A}=\square H y_{, v v} .
$$

An embedding in $E^{5,2}$ is given ${ }^{6}$ by

$$
\begin{aligned}
& y^{1}=(2 H)^{1 / 2}, \quad y^{2}=2^{-1 / 2}(u-v), \\
& y^{3}=2^{-1 / 2}(u+v), \quad y^{4}=x, \quad y^{5}=y, \\
& y^{6}=(2 H)^{1 / 2} \cos u, \quad y^{7}=(2 H)^{1 / 2} \sin u .
\end{aligned}
$$


It is evident from (7) that $g^{\mu \nu} \nabla_{\mu} \nabla_{\nu} y^{A}=0$ $(A=1, \ldots, 7)$, while $G^{\mu \nu}=0$ if and only if $\square H=0$. This last condition defines the subclass $C_{0}$. The rest of the spacetimes in the class $C$ are therefore interpreted in two different ways in the two theories. As solutions of the Einstein-Maxwell equations they represent plane-fronted electromagnetic and gravitational waves propagating in the same direction without dispersion or diffusion, while as solutions of the new-embedding-model equations they represent purely "gravitational" plane-fronted waves.

However, for some other spacetimes in $C$ there are embeddings, still in $E^{5,2}$, which do not satisfy the new-embedding-model vacuum equations. For example, if $H(x, y, u)=\frac{1}{2} x^{2}$, then such ar embedding is given by

$$
\begin{aligned}
& y^{1}=\alpha(v), \quad y^{2}=2^{-1 / 2}(u-v)+\beta(v), \\
& y^{3}=2^{-1 / 2}(u+v)+\beta(v), \quad y^{4}=\gamma(v), \\
& y^{5}=y, \quad y^{6}=x \cos u, \quad y^{7}=x \sin u,
\end{aligned}
$$

where $d \beta / d v=2^{-3 / 2}\left[(d \alpha / d v)^{2}-(d \gamma / d v)^{2}\right]$, and at least one of the otherwise arbitrary functions $\alpha$, $\beta$, and $\gamma$ is nonlinear. This is one example of the class $C_{1}$, and others may easily be constructed.

It may be inferred from our remark (c) above that only trivial modifications of these examples are needed to increase $m$ to 10 . The dependence on the embedding gauge therefore makes it difficult to regard the present new embedding model as an interesting theory of gravitation. Admittedly we have found no manifest conflict with observation, such as a spherically symmetric static solution yielding the wrong perihelion motion, but we contend that the gauge dependence is nevertheless unacceptable.

We have not found any satisfactory way out of this difficulty. Regge and Teitelboim suggest the ad hoc addition of further field equations which make the new embedding model equivalent to Einstein's theory. A more satisfactory alternative would be to find another action functional $I(y)$ whose Euler-Lagrange equations (4) were equivalent to Einstein's equations, but neither they nor we have been able to do this. If it were possible to find an action which removed the excessive gauge freedom, even though the field equations were not equivalent to Einstein's equations, this too might resolve the difficulty. However, one final example suggests that the linearization instability described earlier may impede any easy resolution: Einstein's equations with cosmological constant

$$
G^{\mu \nu}+\lambda g^{\mu \nu}=0
$$

are not satisfied by the flat spacetime metric $g_{\mu \nu}$ $=\eta_{\mu \nu}$. Yet the corresponding new-embedding-model equations

$$
\left(G^{\mu \nu}+\lambda g^{\mu \nu}\right) \nabla_{\mu} \nabla_{\nu} y^{A}=0
$$

are trivially satisfied by

$$
g_{\mu \nu}=\eta_{\mu \nu}, \quad y^{A}=x^{A}, \quad A=1,2,3,4
$$

and by a variety of supernumerary $y$ 's.
*On leave from Brandeis University, Waltham, Massachusetts, U.S.A.; work supported in part by the National Science Foundation, the Science Research Council, and NATO.

${ }^{1} \mathrm{~T}$. Regge and C. Teitelboim, in Proceedings of the Marcel Grossman meeting, Trieste, 1975 (unpublished).

${ }^{2}$ C. Aragone and S. Deser, Nucl. Phys. B96, 327 (1975).

${ }^{3}$ C. J. S. Clarke, Proc. R. Soc. London $\underline{\mathrm{A314}}, 417$ (1970).
${ }^{4}$ M. Janet, Ann. Soc. Polon. Math $\underline{5}, 38$ (1926); E. Cartan, ibid. 6,1 (1927).

${ }^{5} \mathrm{~J}$. Ehlers and W. Kundt, in Gravitation: an introduction to current research, edited by Witten (Wiley, New York, 1962).

${ }^{6} J$. Rosen, Rev. Mod. Phys. 37, 204 (1965). It has been shown by R. Penrose, Rev. Mod. Phys. 37, 215 (1965), that no global embedding in $E^{p, q}$ with $q($ or $p)=1$ is possible. 